\title{
Circumbinary molecular rings around young stars in Orion
}

\author{
L. A. Zapata ${ }^{1,2,3}$, P. T. P. Ho ${ }^{3,4}$, L. F. Rodríguez ${ }^{2}$, P. Schilke ${ }^{1}$, and S. Kurtz ${ }^{2}$ \\ 1 Max-Planck-Institut für Radioastronomie, Auf dem Hügel 69, 53121 Bonn, Germany \\ e-mail: lzapata@mpifr-bonn.mpg.de \\ ${ }^{2}$ CRyA, Universidad Nacional Autónoma de México, Apdo. Postal 3-72 (Xangari), 58089 Morelia, Michoacán, México \\ 3 Harvard-Smithsonian Center for Astrophysics, 60 Garden Street, Cambridge, MA 02138, USA \\ 4 Academia Sinica Institute of Astronomy and Astrophysics, Taipei, Taiwan \\ Received 1 May 2007 / Accepted 6 July 2007
}

\section{ABSTRACT}

\begin{abstract}
We present high angular resolution $1.3 \mathrm{~mm}$ continuum, methyl cyanide molecular line, and $7 \mathrm{~mm}$ continuum observations made with the Submillimeter Array and the Very Large Array, toward the most highly obscured and southern part of the massive star forming region OMC1S located behind the Orion Nebula. We find two flattened and rotating molecular structures with sizes of a few hundred astronomical units suggestive of circumbinary molecular rings produced by the presence of two stars with very compact circumstellar disks with sizes and separations of about 50 AU, associated with the young stellar objects 139-409 and 134-411. Furthermore, these two circumbinary rotating rings are related to two compact and bright hot molecular cores. The dynamic mass of the binary systems obtained from our data are $\geq 4 M_{\odot}$ for 139-409 and $\geq 0.5 M_{\odot}$ for 134-411. This result supports the idea that intermediate-mass stars will form through circumstellar disks and jets/outflows, as the low mass stars do. Furthermore, when intermediate-mass stars are in multiple systems they seem to form a circumbinary ring similar to those seen in young, multiple low-mass systems (e.g., GG Tau and UY Aur).
\end{abstract}

Key words. ISM: HII regions - ISM: jets and outflows - ISM: molecules - stars: formation - instrumentation: high angular resolution

\section{Introduction}

About thirty years ago a new major puzzle emerged in the field of stellar astrophysics: How do massive stars form?, where massive stars are those with more than $10 M_{\odot}($ Kahn 1974; Larson \& Starrfield 1971; Yorke \& Kruegel 1977). It was believed that the powerful radiation fields and stellar winds produced at the very beginning of their lives will inhibit accretion of material limiting their mass growth to about $10 M_{\odot}$. In the two last decades, several alternatives have been proposed to solve this puzzle. Among the most important are the formation of massive stars through dense disks and jets/outflows (Nakano 1989; Jijina \& Adams 1996), in very dense and turbulent cores (McKee \& Tan 2002), through the merging of smaller stars (Bonnell et al. 1998), and through ionized accretion flows that forms an ionized disk or torus around a group of stars (Sollins et al. 2005; Keto \& Wood 2006). Discriminating between these alternatives remains an observational challenge (Cesaroni et al. 2007).

OMC1S or Orion-S is the "twin" dusty massive molecular core of the Orion BN-KL core. It is located almost at the same angular distance from the "Trapezium" as Orion BN-KL $\left(\sim 1^{\prime}\right)$, but to the southwest of the former. We adopt a distance of $460 \mathrm{pc}$ to the Orion Nebula (Bally et al. 2000). The OMC1S region has a mass of about $100 M_{\odot}$, similar to that reported for BN-KL (Mezger et al. 1990), but with a bolometric luminosity of $\sim 10^{4} L_{\odot}$, which is a factor of 10 less (Mezger et al. 1990; Drapatz et al. 1983). This difference in luminosity might be attributed to OMC1S being less evolved than Orion BN-KL, as inferred if one compares the molecular line emission from both regions (McMullin et al. 1993). With time, the massive stars forming in OMC1S might reach their final masses and shine with much larger luminosity than now. This possible evolutionary scheme has been also suggested to be taking place in the NGC 6334I region (Rodríguez et al. 2007).

In this letter we report for the first time the possible presence of two circumbinary molecular rotating rings located in the OMC1S region with sizes of a few hundred Astronomical Units (AU) around two very compact circumstellar disks and that are associated with intermediate-mass (proto)stars.

\section{Observations}

The observations were made with the Submillimeter Array (SMA) ${ }^{1}$ and the Very Large Array (VLA) ${ }^{2}$ during 2004 September 2 and November 10, respectively.

The SMA was in its extended configuration, which includes 21 independent baselines ranging in projected length from 16 to $180 \mathrm{~m}$. The phase reference center of the observations was $\mathrm{RA}=05^{\mathrm{h}} 35^{\mathrm{m}} 14^{\mathrm{s}}$, Dec $=-05^{\circ} 24^{\prime} 00^{\prime \prime}(\mathrm{J} 2000.0)$. The receivers were tuned at a frequency of $230.534 \mathrm{GHz}$ in the upper sideband (USB), while the lower sideband (LSB) was centered at $220.53 \mathrm{GHz}$. The $\mathrm{CH}_{3} \mathrm{CN}\left[12_{4}-11_{4}\right]$ transition was detected in the LSB at a frequency of $220.679 \mathrm{GHz}^{3}$. The full bandwidth of the SMA correlator is $4 \mathrm{GHz}$ ( $2 \mathrm{GHz}$ in each band). The SMA

\footnotetext{
1 The Submillimeter Array is a joint project between the Smithsonian Astrophysical Observatory and the Academia Sinica Institute of Astronomy and Astrophysics, and is funded by the Smithsonian Institution and the Academia Sinica.

2 The National Radio Astronomy Observatory is a facility of the National Science Foundation operated under cooperative agreement by Associated Universities, Inc.

${ }^{3}$ Several hyperfine transitions of the $\mathrm{CH}_{3} \mathrm{CN}[12-11]$ molecule were detected toward these sources, however, the images were made from this transition.
} 


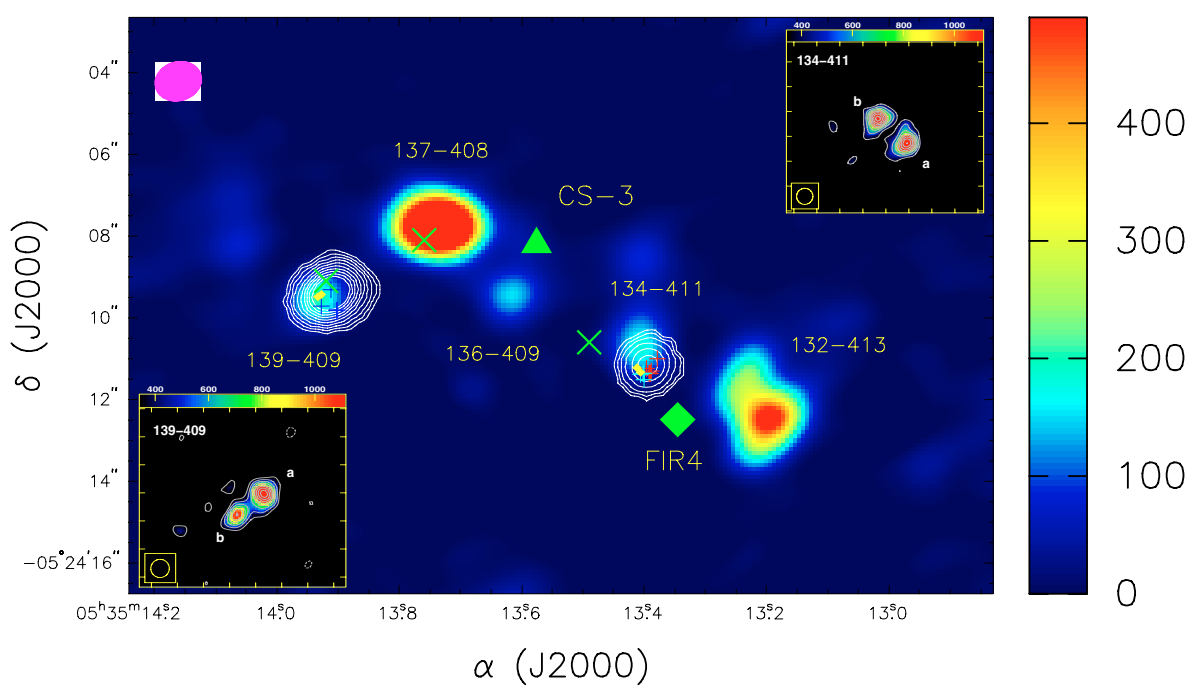

Fig. 1. SMA $1.3 \mathrm{~mm}$ continuum color image of the southern most region of OMC1S (from Zapata et al. 2005), overlaying on the $\mathrm{CH}_{3} \mathrm{CN}\left[12_{4}-11_{4}\right]$ integrated molecular emission of the hot molecular cores 139-409 and 134-411 (white contours). The contours of the $\mathrm{CH}_{3} \mathrm{CN}\left[12_{4}-11_{4}\right]$ integrated molecular emission are $-5,5,10,15,20,25,30,35,40,45$ and 50 times $120 \mathrm{mJy}$ beam $^{-1} \mathrm{~km} \mathrm{~s}^{-1}$, the rms noise of the image. The integration is over a velocity range of -5 to $17 \mathrm{~km} \mathrm{~s}^{-1}$. The millimeter sources $139-409,134-411,132-413,135-409$ and $137-408$ were reported for the first time by Zapata et al. (2005). The synthesized beam of the $\mathrm{CH}_{3} \mathrm{CN}$ image is $1.13^{\prime \prime} \times 0.93^{\prime \prime}$ with a PA $=-73^{\circ}$ and it is shown in the upper left corner. The scale bar indicates the $1.3 \mathrm{~mm}$ continuum emission in $\mathrm{mJy} \mathrm{beam}^{-1}$. The yellow rhombi indicate the positions of the $7 \mathrm{~mm}$ continuum compact radio binaries. The green rhombus and triangle denote the position of the source FIR 4 (Mezger et al. 1990) and the millimeter source CS 3 (Mundy et al. 1986), respectively. The blue and red crosses indicate the position of the blue- and red-shifted $\mathrm{H}_{2} \mathrm{O}$ maser spots, respectively, reported by Gaume et al. (1998). Note that the masers associated with the hot molecular core (134-411) show a large velocity gradient, going from -20 to $+45 \mathrm{~km} \mathrm{~s}^{-1}$. The green " $\mathrm{X}$ " symbols indicate the position of the $3 \mathrm{~mm}$ BIMA continuum sources reported by Eisner \& Carpenter (2006). In the left bottom and right upper corners we show the $7 \mathrm{~mm}$ continuum compact radio binaries located on the centers of the hot cores 139-409 and 134-411. The scale bar indicates the $7 \mathrm{~mm}$ continuum emission in $10^{-3} \mathrm{mJy}_{\text {beam }}{ }^{-1}$ on both images. In the left bottom corner box the contours are $-2,2,3,4,5,6,7,8,9$ times $0.15 \mathrm{mJy}_{\text {beam }}^{-1}$, the rms noise of the image. In the right upper corner box the contours are $-2,2,3$, $4,5,6,7,8,9$ times $0.15 \mathrm{mJy}^{\text {beam }}{ }^{-1}$ the rms noise of the image. The synthesized beam is shown in the bottom left corner of each box. The sizes of these boxes are $0.6^{\prime \prime} \times 0.6^{\prime \prime}$.

digital correlator was configured in spectral windows ("chunks") of $104 \mathrm{MHz}$ each, with 32 channels distributed over each spectral window, providing a resolution of $3.25 \mathrm{MHz}\left(4.29 \mathrm{~km} \mathrm{~s}^{-1}\right)$ per channel.

The zenith opacity measured $\left(\tau_{230 \mathrm{GHz}}\right)$ with the NRAO tipping radiometer located at the Caltech Submillimeter Observatory was $\sim 0.04$, indicating very good weather conditions during the experiment. Observations of Callisto provided the absolute scale for the flux density calibration. The phase and amplitude calibrators were the quasars $0423-013$ and $3 \mathrm{C} 120$, with measured flux densities of $2.343 \pm 0.006$ and $0.563 \pm 0.003 \mathrm{Jy}$, respectively. The absolute flux density calibration uncertainty is estimated to be $20 \%$, based on SMA monitoring of quasars. Further technical descriptions of the SMA and its calibration schemes are found in Ho et al. (2004).

The VLA was in its most extended A-configuration, providing baselines with a maximum projected length of $36 \mathrm{~km}$. The central frequency observed was $43.34 \mathrm{GHz}$. The absolute amplitude calibrator was $1331+305$ (with an adopted flux density of $1.45 \mathrm{Jy}$ ) and the phase calibrator was 0541-056 (with a bootstrapped flux density of $1.10 \pm 0.09$ ). The phase center of these observations was the same as for the SMA observations.

The SMA data were calibrated using the $\mathrm{MIR}^{4}$ software package, while the VLA data were calibrated using the AIPS software. The calibrated data were imaged and analyzed in the standard manner using the MIRIAD and AIPS packages. We used the ROBUST parameter set to 0, for an optimal compromise between sensitivity and angular resolution. The resulting

${ }_{4}$ The MIR cookbook by C. Qi can be found at http://cfa-www.harvard. edu/ cqi/mircook.html continuum image rms at $7 \mathrm{~mm}$ was $0.15 \mathrm{mJy}^{-1}$ beam $^{-1}$, at an angular resolution of 0 ' $^{\prime} 05 \times 00^{\prime} 04$ with a PA $=-6^{\circ}$. For the SMA observations the line image rms noise was $120 \mathrm{mJy}^{\text {beam }}{ }^{-1}$ for each channel at an angular resolution of 1. .' $13 \times 0$.' 93 with a $\mathrm{PA}=-73^{\circ}$.

\section{Results and discussion}

\section{1. $1 \mathrm{~mm}$ continuum and molecular emission}

In Fig. 1, we have overlayed the SMA $1.3 \mathrm{~mm}$ continuum image from Zapata et al. (2005), with the integrated $\mathrm{CH}_{3} \mathrm{CN}\left[12_{4}-11_{4}\right]$ emission in the southern, most obscured part of OMC1S. Furthermore, we have also included on this image the positions of the water maser spots from Gaume et al. (1998), the positions of the strong $3 \mathrm{~mm}$ continuum and FIR sources located here (Mezger et al. 1990; Mundy et al. 1986; Eisner \& Carpenter 2006), and the positions of the $7 \mathrm{~mm}$ binary systems associated with the sources 139-409 and 134-411.

In this image, we can see five $1.3 \mathrm{~mm}$ continuum sources (139-409, 137-408, 136-409, 134-411, and 132-413), but with only two sources (139-409 and 134-411) showing very compact $\mathrm{CH}_{3} \mathrm{CN}\left[12_{4}-11_{4}\right]$ emission. Some of these continuum sources (139-409, 137-408, 134-411 and 132-413) are the $1.3 \mathrm{~mm}$ counterparts of the $3.6 \mathrm{~cm}, 1.3 \mathrm{~cm}$ and $3 \mathrm{~mm}$ sources reported by Eisner \& Carpenter (2006) and Zapata et al. (2004a,b) and which are interpreted as UC HII regions, ionized thermal jets and/or massive circumstellar disks. The 139-409 and 134-411 sources show strong compact molecular emission also in different lines (e.g. series of $\mathrm{CH}_{3} \mathrm{CN}, \mathrm{CH}_{3} \mathrm{OH}, \mathrm{SO}_{2},{ }^{34} \mathrm{SO}, \mathrm{H}_{2} \mathrm{CO}$, etc), and are 

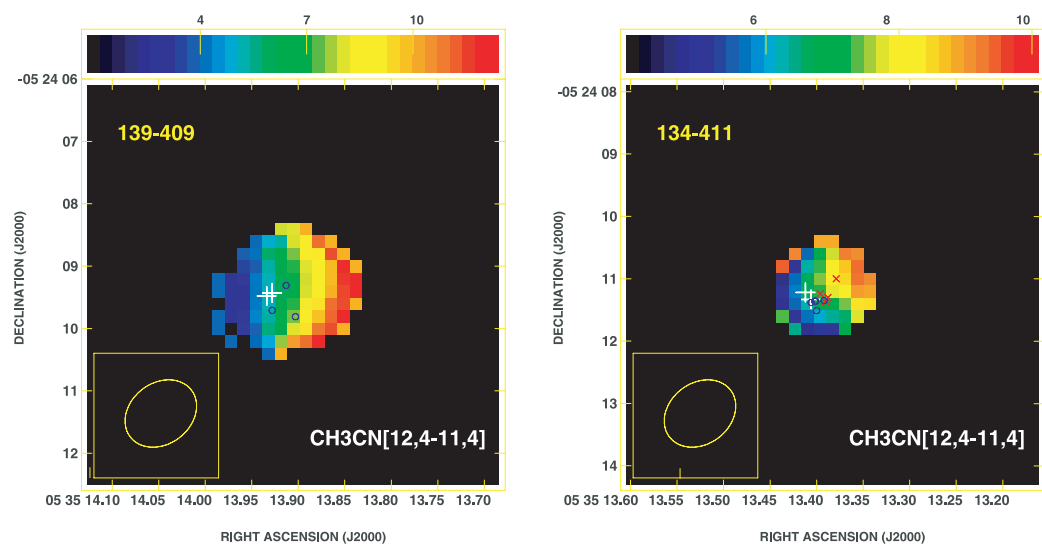

Fig. 2. $\mathrm{CH}_{3} \mathrm{CN}\left[12_{4}-11_{4}\right]$ first moment maps of the molecular hot cores $139-409$ and 134-411. The white crosses indicate the positions of the $7 \mathrm{~mm}$ compact radio binaries shown in Fig. 1. The systemic LSR radial velocity of the ambient molecular cloud is about $7 \mathrm{~km} \mathrm{~s}^{-1}$. The scale bar indicates the LSR radial velocities of the core in $\mathrm{km} \mathrm{s}^{-1}$. Note that both cores show rotation of a few kilometers per second, with different orientations. The synthesized beam is shown in the bottom left corner of each image. The spectral resolution is $4 \mathrm{~km} \mathrm{~s}^{-1}$. Note that our spectral resolution is comparable with the linewidth of the $\mathrm{CH} 3 \mathrm{CN}$ transition. The blue circles and red crosses indicate the position of the blue- and red-shifted $\mathrm{H}_{2} \mathrm{O}$ masers, as in Fig. 1.

thus related with two bright, very compact hot molecular cores associated with intermediate-mass stars (Zapata et al., in prep.).

The $\mathrm{CH}_{3} \mathrm{CN}\left[12_{4}-11_{4}\right]$ line molecular emission associated with the source 139-409 has a deconvolved size of $0 . ' 64 \pm 0 .{ }^{\prime} 03 \times$ $0.45 \pm 0 . \prime 04$ (or $294 \mathrm{AU} \pm 14 \mathrm{AU} \times 207 \mathrm{AU} \pm 18 \mathrm{AU}$ ) with a PA of $87^{\circ} \pm 7^{\circ}$, while the molecular emission associated with the source 134-411 has dimensions of $0.36 \pm 0.0^{\prime} 07 \times \leq 0.33$ (or $166 \mathrm{AU} \pm 32 \mathrm{AU} \times \leq 151 \mathrm{AU}$ ) with a PA of $116^{\circ} \pm 10^{\circ}$.

The $\mathrm{CH}_{3} \mathrm{CN}\left[12_{4}-11_{4}\right]$ molecular emission shows a total velocity shift of $5 \mathrm{~km} \mathrm{~s}^{-1}$ for the object $139-409$ and of $2.5 \mathrm{~km} \mathrm{~s}^{-1}$ for the object 134-411 and if one assumes that this molecular gas is rotating in a Keplerian way and uses the deconvolved sizes of the molecular structures presented above, we calculated a lower limit for the mass of the central objects shown in Table 1. Since these are lower limits, we think that the binaries at the center of these circumstellar disks are formed by intermediate-mass stars. The velocity gradients in both objects were determined from a simultaneous Gaussian fitting to the $K=0$ to 6 components of the $\mathrm{CH}_{3} \mathrm{CN}[12-11]$ transition. Finally, the hypothesis of rotation is suggested by the observation that the major axis of the molecular structures seems to be approximately aligned with the direction of the velocity gradients.

\section{2. $7 \mathrm{~mm}$ continuum emission}

The sources $139-409$ and $134-411$ in the $7 \mathrm{~mm}$ continuum observations were resolved into two compact binary systems with separations and sizes of about $50 \mathrm{AU}$ (see Fig. 1) that are interpreted as very compact circumstellar disks. The small size of these disks might be explained as tidally truncated disks as those observed in the L1551 IRS5 system (Rodríguez et al. 1998). In the case of 134-411 the orientation of the $\mathrm{H}_{2} \mathrm{O}$ masers is very close to that observed for the circumbinary molecular disk (see Fig. 2), suggesting that maybe these masers are either associated with it. However, the velocity gradient observed in the masers is too large to be explained in terms of Keplerian rotation.

From the millimeter observations of Zapata et al. (2005) and the $7 \mathrm{~mm}$ continuum observations presented here, we calculate that the sources 139-409 and 134-411 have average spectral indices of $2.6 \pm 0.3$ and $2.7 \pm 0.3$, respectively. We then suggest that at 7 and $1.3 \mathrm{~mm}$ we are observing optically thin dust emission with a dust mass opacity coefficient that varies with frequency
Table 1. Physical parameters of the circumbinary rings.

\begin{tabular}{|c|c|c|c|c|c|c|}
\hline \multirow[b]{2}{*}{ Name } & \multirow[b]{2}{*}{$\begin{array}{c}\text { RA } \\
{[\mathrm{J} 2000]}\end{array}$} & \multirow[b]{2}{*}{$\begin{array}{c}\text { Dec } \\
{[\mathrm{J} 2000]}\end{array}$} & \multirow{2}{*}{$\begin{array}{c}\text { Deconv. } \\
\text { Radius } \\
\text { [AU] }\end{array}$} & \multirow{2}{*}{$\begin{array}{c}\text { Rot. } \\
\text { Vel. } \\
{\left[\mathrm{km} \mathrm{s}^{-1}\right]}\end{array}$} & \multicolumn{2}{|c|}{ Mass } \\
\hline & & & & & $\begin{array}{l}\text { Dyn. } \\
{\left[M_{\odot}\right]}\end{array}$ & $\begin{array}{l}\mathrm{Gas}^{a} \\
{\left[M_{\odot}\right]}\end{array}$ \\
\hline $139-409$ & 053513.912 & -052409.40 & $147 \pm 7$ & 5 & $\geq 4$ & 0.08 \\
\hline $134-411$ & 053513.394 & -052411.15 & $83 \pm 16$ & 2.5 & $\geq 0.5$ & 0.12 \\
\hline \multicolumn{7}{|c|}{ The circumstellar disks } \\
\hline Name & $\begin{array}{c}\mathrm{RA} \\
{[\mathrm{J} 2000]}\end{array}$ & $\begin{array}{c}\text { Dec } \\
{[\mathrm{J} 2000]}\end{array}$ & $\begin{array}{c}\text { Flux } \\
\text { Density } \\
{[\mathrm{mJy}]}\end{array}$ & $\begin{array}{c}\text { Undeconv. } \\
\text { Radius } \\
\text { [AU] }\end{array}$ & $\begin{array}{r}\text { Mas } \\
{[} \\
\end{array}$ & $\begin{array}{l}\text { of the } \\
\text { as }^{a} \\
\left.\mu_{\odot}\right]\end{array}$ \\
\hline $139-409 a$ & 053513.928 & -052409.41 & $2.5 \pm 0.5$ & 25 & & 05 \\
\hline $139-409 b$ & 053513.933 & -05 2409.47 & $3.2 \pm 0.5$ & 20 & & 06 \\
\hline $134-411 \mathrm{a}$ & 053513.406 & -052411.33 & $3.3 \pm 0.5$ & 25 & & 06 \\
\hline $134-411 b$ & 053513.412 & -052411.22 & $3.2 \pm 0.5$ & 25 & & 06 \\
\hline
\end{tabular}

(a) The masses of the gas were obtained assuming a dust temperature value of $100 \mathrm{~K}$, an adopted value of $\kappa_{1.3 \mathrm{~mm}}=1.5 \mathrm{~cm}^{2} \mathrm{~g}^{-1}$ (the average of the values of $1.0 \mathrm{~cm}^{2} \mathrm{~g}^{-1}$, valid for grains with thick dust mantles, and $2.0 \mathrm{~cm}^{2} \mathrm{~g}^{-1}$, valid for grains without mantles) and the flux densities at $1.3 \mathrm{~mm}$ of $180 \mathrm{mJy}$ for 139-409 and of $270 \mathrm{mJy}$ for 134-411.

as $\kappa \propto v^{0.6}$ and $\kappa \propto v^{0.7}$ (suggesting grain growth). With this information we can estimate the enclosed masses of the $7 \mathrm{~mm}$ sources. Assuming optically thin, isothermal dust emission and a gas-to-dust ratio of 100 (Sodroski et al. 1997) and an adopted value of $\kappa_{1.3 \mathrm{~mm}}=1.5 \mathrm{~cm}^{2} \mathrm{~g}^{-1}$ (and then $\kappa_{7} \mathrm{~mm}$ equal to 0.46 for $139-409$ and $0.54 \mathrm{~cm}^{2} \mathrm{~g}^{-1}$ for 134-411), and a typical dust temperature value of $100 \mathrm{~K}$, we derive total enclosed masses for those putative circumstellar disks that are shown in Table 1. From the results of this table we conclude that the masses of these systems are dominated by the stellar components, with the disks contributing with masses of order $0.1 M_{\odot}$. We also note that the mass associated with the circumbinary disks is comparable with that associated with the circumstellar disks (see Table 1).

\subsection{Two circumbinary molecular rings?}

Since the $\mathrm{CH}_{3} \mathrm{CN}$ molecule is a high-density tracer (Cesaroni et al. 1999), the elongated structures traced by this molecule in 139-409 and 134-411 suggest two flattened circumbinary molecular rings observed in nearly edge-on. Moreover, the first 


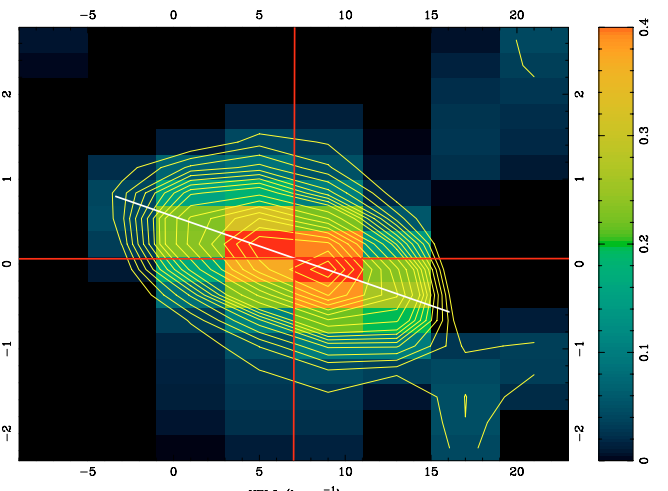

VELO $\left(\mathrm{km} \mathrm{s}^{-1}\right)^{1}$

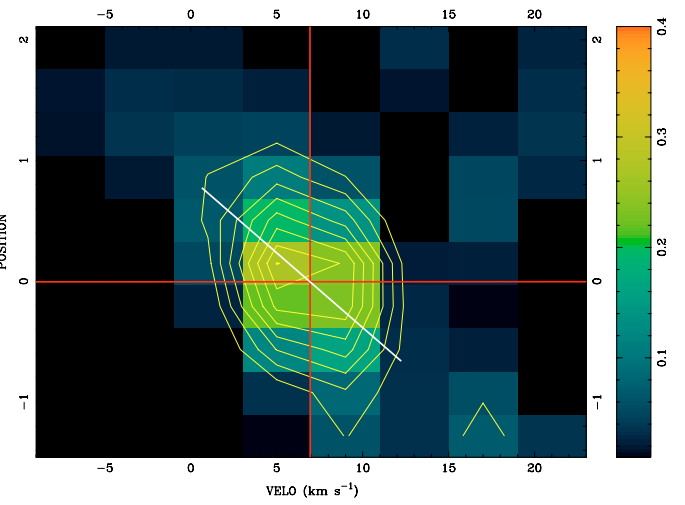

Fig. 3. Position-velocity diagrams of the $\mathrm{CH}_{3} \mathrm{CN}\left[12_{4}-11_{4}\right]$ molecular emission of 139-409 (left) and 134-411 (right) computed along the directions with $\mathrm{PA}=87^{\circ}$ and $\mathrm{PA}=115^{\circ}$, respectively. The velocity and angular resolutions are $4 \mathrm{~km} \mathrm{~s}^{-1}$ and $\sim 1^{\prime \prime}$, respectively. The horizontal lines indicate the position of the center of each source, while the vertical lines indicate the systemic velocity of the cloud. The systemic LSR radial velocity of the ambient molecular cloud is about $7 \mathrm{~km} \mathrm{~s}^{-1}$. Note the velocity gradients shown by the white lines. In the top image the contours are $2,3,4,5,6,7,8,9,10,12,14,16,18,20,22$, and 24 times $120 \mathrm{mJy}$ beam $^{-1}$, the rms noise of the image. In the bottom image the contours are $2,3,4,5,6,7,8,9$, and 10 times $120 \mathrm{mJy} \mathrm{beam}^{-1}$, the rms noise of the image. Note that our spectral resolution is comparable with the linewidth of the $\mathrm{CH}_{3} \mathrm{CN}$ transition.

moment of the $\mathrm{CH}_{3} \mathrm{CN}$ [12-11] emission shows a total velocity gradient of about a few kilometers per second that seems to be aligned with the major axes of both structures, supporting also this interpretation (see Fig. 2). We note that the two $7 \mathrm{~mm}$ sources are aligned along the velocity gradient in 139-409, but perpendicular to it in 134-411. One might speculate that the former ring is almost edge-on, whereas the latter could be significantly inclined with respect to the line of sight. In fact, if the binary system is coplanar with the ring and seen edge-on, the two stars must appear along the direction of the velocity gradient; instead, if the ring is inclined, the projections of the two stars on the plane of the sky may appear along any direction. The fact that the velocity gradient is much less in 134-411 than in 139-409 is consistent with this interpretation. Finally, in Fig. 3 we show that the kinematics of the molecular gas in both cores, as computed along the major axes, seems to be consistent with a "rigid body law". This kinematic behavior also suggests that the molecular gas in both flattened structures seems to be only in a rotating ring.

Those molecular ring-shaped circumbinary structures have been also observed in low-mass young stars, e.g. GG Tau and UY Aur (Guilloteau et al. 1999; Duvert et al. 1998). This kind of structures appear to be tidally stimulated during the formation of binary or multiple systems of stars at its center. When a multiple system is formed the very compact circumstellar disks associated with the protostars clean its vicinity (or the innermost part of the large circumbinary disk) forming thus a circumbinary ring around them (Mathieu et al. 2000). This circumbinary ring might not transfer material to the compact circumstellar disks anymore, however, see Monin et al. (2006) for a more detail discussion of this phenomenon.

It is interesting to note that much larger molecular rotating structures have also been observed around multiple OB (proto)stars (Torrelles et al. 1983; Beltrán et al. 2005; Sollins et al. 2005). However, contrary to these circumbinary rings, these molecular structures (also known as "toroids") seem to be transient and in non-gravitational-equilibrium (Beltrán et al. 2005).

In conclusion, we interpret the flattened molecular structures associated with the sources 139-409 and 134-411 as two circumbinary rings around binary systems traced by very compact $7 \mathrm{~mm}$ circumstellar disks associated with young intermediatemass (proto)stars. However, we believe that more observations with higher angular and spectral resolution are necessary to confirm our interpretation. Finally, this result supports also the idea that intermediate-mass stars form through circumstellar disks and jets/outflows, as the low mass stars do.

Acknowledgements. We thank the anonymous referee for many valuable suggestions. L.F.R. acknowledges the support of CONACyT, México and DGAPA, UNAM. Facilities: SMA and VLA

\section{References}

Bally, J., O’Dell, C. R., \& McCaughrean, M. J. 2000, AJ, 119, 2919 Beltrán, M. T., Cesaroni, R., Neri, R., et al. 2005, A\&A, 435, 901 Bonnell, I. A., Bate, M. R., \& Zinnecker, H. 1998, MNRAS, 298, 93 Cesaroni, R., Felli, M., Jenness, T., et al. 1999, A\&A, 345, 949

Cesaroni, R., Galli, D., Lodato, G., Walmsley, C. M., \& Zhang, Q. 2007, in Protostars and Planets V, ed. B. Reipurth, D. Jewitt, \& K. Keil (Tucson: University of Arizona Press), 197

Drapatz, S., Haser, L., Hofmann, R., Oda, N., \& Iyengar, K. V. K. 1983, A\&A, 128,207

Duvert, G., Dutrey, A., Guilloteau, S., et al. 1998, A\&A, 332, 867

Eisner, J. A., \& Carpenter, J. M. 2006, ApJ, 641, 1162

Gaume, R. A., Wilson, T. L., Vrba, F. J., et al. 1998, ApJ, 493, 940

Guilloteau, S., Dutrey, A., Simon, M., et al. 1999, A\&A, 348, 570

Ho, P. T. P., Moran, J., \& Lo, K. Y. 2004, ApJ, 616, L1

Jijina, J., \& Adams, F. 1996, ApJ, 462, 87

Kahn, F. 1974, A\&A, 37, 149

Keto, E., \& Wood, K. 2006, ApJ, 637, 850

Larson, R. B., \& Starrfield, S. 1971, A\&A, 13, 190

Mathieu, R. D., Ghez, A. M., Jensen, E. L. N., \& Simon, M. 2000, Protostars and Planets IV, ed. V. Mannings, A. P. Boss, \& S. S. Russell (University of Arizona Press), 703

Monin, J.-L., Clarke, C. J., Prato, L., \& McCabe, C. 2006, in Protostars and Planets V, ed. B. Reipurth, D. Jewitt, \& K. Keil (Tucson: University of Arizona Press), 197

McKee, C. F., \& Tan, J. C. 2002, Nature, 416, 59

McMullin, J. P., Mundy, L. G., \& Blake, G. A. 1993, ApJ, 405, 599

Mezger, P. G., Zylka, R., \& Wink, J. 1990, A\&A, 228, 95

Nakano, T. 1989, ApJ, 345, 464

Sollins, P. K., Zhang, Q., Keto, E., \& Ho, P. T. P. 2005, ApJ, 631, 399

Rodríguez, L. F., D’Alessio, P., Wilner, D. J., et al. 1998, Nature, 395, 355

Rodríguez, L. F., Zapata, L. A., \& Ho, P. T. P. 2007, ApJ, 654, L143

Torrelles, J. M., Rodríguez, L. F., Canto, J., et al. 1983, ApJ, 274, 214

Yorke, H. W., \& Kruegel, E. 1977, A\&A, 54, 183

Zapata, L. A., Rodríguez, L. F., Kurtz, S. E., \& O’Dell, C. R. 2004a, AJ, 127, 2252

Zapata, L. A., Rodríguez, L. F., Kurtz, S. E., O’Dell, C. R., \& Ho, P. T. P. 2004b, ApJ, 610, L121 